\title{
Time reversal symmetry and the difference between relaxations and building-up periods
}

\author{
Philipp G. Meyer $\odot^{*}$ and Holger Kantz $\odot$ \\ Max Planck Institute for the Physics of Complex Systems, Dresden D-01187, Germany
}

(Received 31 March 2021; accepted 22 July 2021; published 11 August 2021)

\begin{abstract}
Autocorrelations in stationary systems are time-symmetric, irrespective of the signal's properties. Linear dynamics is usually associated with signals which are statistically time inversion invariant. This is known to be broken for non-Gaussian models. In this paper, we develop a theoretical framework of time reversibility of linear models based on noncontinuous driving. We identify the inverse decay exponent of the autocorrelation function as either a characteristic time for the building-up of extreme events in the time series or of relaxations after these extreme events. If the characteristic time is known, the dynamics can be inverted in both directions in time and the residuals can be compared, which gives a criterion for the type of time inversion asymmetry. The method is applied to two time series from atmospheric science with different behavior.
\end{abstract}

DOI: 10.1103/PhysRevE.104.024208

\section{INTRODUCTION}

For many systems of high complexity, accurate modeling of the physical process is very challenging. In such cases, and in particular, if the main interest does not lie in the understanding of the physics behind it, there is a demand for data models that reproduce the statistics of the system without requiring physical insight into the dynamics. The most prominent properties to be reproduced accurately are the autocorrelations and the probability distribution. However, these properties do not completely describe the dynamics. They do not even uniquely define a stochastic process. According to the Kolmogorov extension theorem [1], the set of all multivariate joint probability distributions would be needed, not only the one-point (i.e., marginal) and two-point distributions.

One property that is neither captured by the autocorrelations nor the distribution is the time-reversal symmetry. The microscopic theories in physics are symmetric in time, however, macroscopic theories are not, in general, as the concept of entropy very clearly shows. The difficulty is to define an observable which indicates this property. Measures can be based on the symmetry of the increment distribution [2], on higher-order correlations [3], or from ordinal patterns [4]. The theoretical requirements for dynamical systems with this property were previously discussed [5]. Time asymmetry has the very important implication that all static transformations of linear Gaussian random processes can be excluded as a suitable model for the data [6], and it has been used as a test for nonlinearity in the context of surrogate data $[7,8]$.

\footnotetext{
*philippm@pks.mpg.de
}

Published by the American Physical Society under the terms of the Creative Commons Attribution 4.0 International license. Further distribution of this work must maintain attribution to the author(s) and the published article's title, journal citation, and DOI. Open access publication funded by the Max Planck Society.
Exponential decay of autocorrelations is a very widespread phenomenon. It originates from fundamental physical equations and is related to chaos theory if the second-largest eigenvalue of the Frobenius-Perron operator is inside a disk of the complex plane with radius $<1$ [9]. Here the decay is caused by the mixing of the trajectories in phase space. Also, exponential decay is typical in much simpler dynamics, such as linearly damped systems.

The autoregressive process of order one, $\operatorname{AR}(1)$ [10], is a popular time series model, which describes the pure exponential decay of autocorrelations. It is defined by the iteration relation

$$
X(t)=g X(t-1)+\eta(t) .
$$

Here $g \in[0,1]$ is a damping parameter (we disregard here negative values $g \in[-1,0]$ ), which determines the relaxation time $\tau$. The driving noise $\eta(t)$ is white $\left\langle\eta(t) \eta\left(t^{\prime}\right)\right\rangle=\delta\left(t-t^{\prime}\right)$ with a Gaussian normal probability distribution $(\langle\rangle$ is an ensemble average). The mean of $X$ and $\eta$ is zero, so to apply the model to the data the mean value is subtracted from the time series. The autocorrelation function is given by

$$
C(t)=\exp \left(-\frac{|t|}{\tau}\right) \text { with } \tau=-\frac{1}{\log (g)} .
$$

The AR(1) model is used in statistical analysis of all kinds of systems. Due to its simplicity and physical plausibility, it is often applied as a zero-order model or null hypothesis. Examples include global temperatures [11], wind speed [12], finance [13], engineering [14], and many more.

This paper discusses the property of time-reversal asymmetry from the perspective of autoregressive dynamics. In Sec. II we introduce a model that generalizes the autoregressive process of order one. In Sec. III we show how this model can be used for a hypothesis test for time symmetry of a time series. We also discuss how robust our method is for ill-fitted model parameters, and how the property of time symmetry has to be distinguished from the property of Gaussianity. In Sec. IV, we finally illustrate that the proposed approach gives clear 
(a)
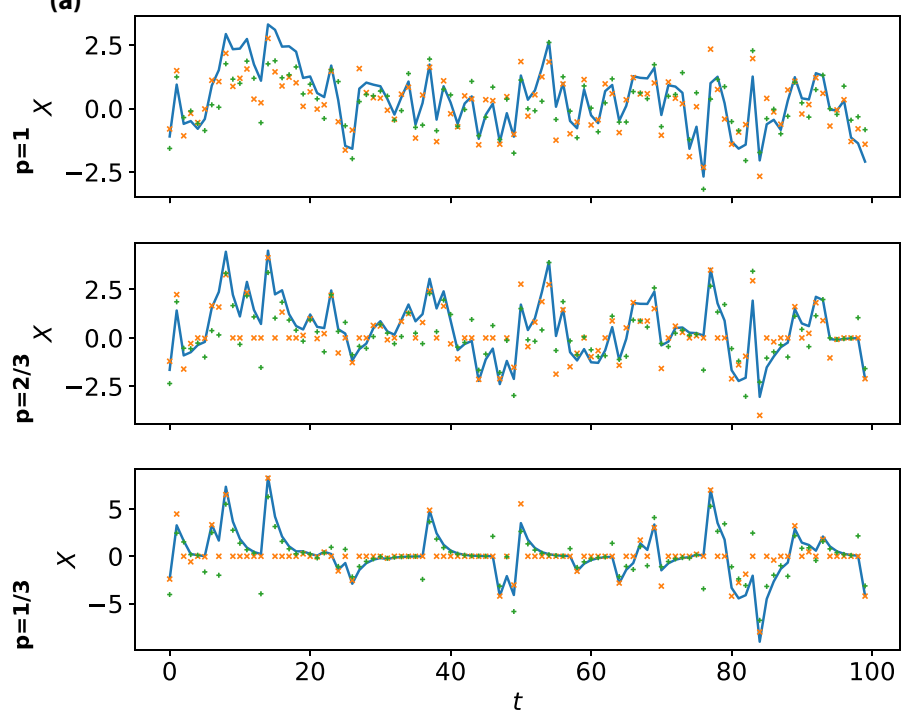

(b)
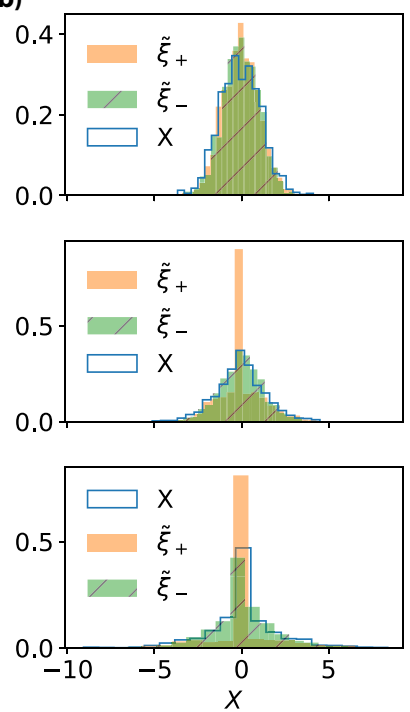

(c)
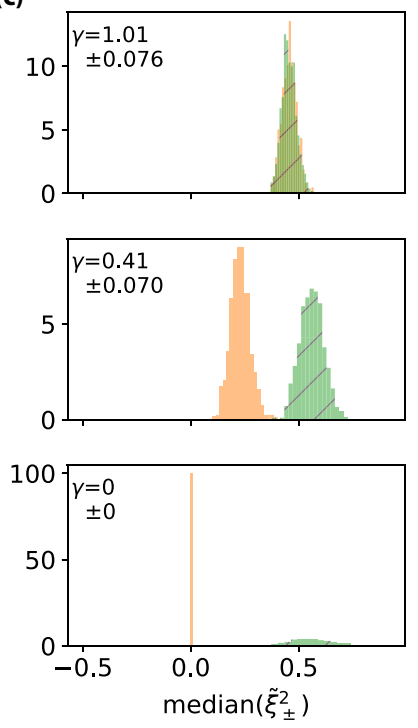

FIG. 1. Generalized AR(1) processes $(g=0.5)$ with length 1000 with $p=1(1), 2 / 3$ (2), and $1 / 3$ (3). (a): Segments of time series (continuous lines) and reconstructed noises $\tilde{\xi}_{+}$(orange 'x') and $\tilde{\xi}_{-}$(green '+'); (b) Histograms of $X$ (blue line), $\tilde{\xi}_{+}$(orange), and $\tilde{\xi}_{-}$(green dashed) of one trajectory. (c) Comparison of median $\left(\tilde{\xi}_{ \pm}^{2}\right)$ for an ensemble of 1000 trajectories. The distributions coincide for $p=1$. For smaller values of $p$ the values become distinguishable.

results for real measured time series. We use data of stratospheric aerosol optical depth, reflecting volcano eruptions, and ${ }^{18} \mathrm{O}$ isotope concentrations in Greenland ice cores, which are proxies for surface temperatures and show the so-called Dansgaard-Oeschger events. The results are discussed and summarized in the Conclusion.

\section{GENERALIZED AR(1) MODEL}

The AR(1) model is a Gaussian process in discrete time. The exponential decay of autocorrelations does not depend on the distribution of the noise $\xi(t)$, but only on the iteration relation (1). So we can generalize the AR(1) model by choosing a different type of uncorrelated noise. It is not even required that the noise $\xi(t)$ has zero mean, as we will see later. One specific way to generate non-Gaussian noise is by not driving the system with a Gaussian random variable at every point in time, but only with a certain probability, and let the process run without driving otherwise: $X(t)=g X(t-1)+\xi(t)$ with

$$
\xi(t)=\left\{\begin{array}{cll}
\frac{1}{p} \eta(t) & \text { with probability } & p, \\
0 & \text { with probability } & 1-p .
\end{array}\right.
$$

This corresponds to introducing a waiting time for the noise driving. The waiting durations are exponentially distributed and the noise is a Gaussian-dressed Poisson process.

In Fig. 1 in the first column we show segments of time series, where the driving noise is continuous with $p=1$ (first row), has waiting periods with $p=2 / 3$ (second row), and with $p=1 / 3$ (third row).

Even for $p<1$, the autocorrelation function is still timesymmetric, however, the process is not. This is very clear to see in the time series with $p=1 / 3$. The spikes appear abruptly due to large values in the driving noise. They disappear only slowly with an exponential relaxation as described by the defining Eq. (1) of the dynamics and the autocorrelation function.

\section{FINDING ASYMMETRIES BY INVERTING AR(1) DYNAMICS}

The noise $\xi(t)$ of the $\mathrm{AR}(1)$ process can be reconstructed by inverting Eq. (1)

$$
\tilde{\xi}(t)=X(t)-g X(t-1) .
$$

We denote the estimated noise the $\tilde{\xi}(t)$ in contrast to the the true driving of the process $\xi(t)$. As pointed out in the previous section, the $\operatorname{AR}(1)$ iteration relation describes spikes and consecutive relaxations. However, the autocorrelation function, as well as the probability distribution of this process, is identical to those of a process with an exponential building-up, with statistical resetting to zero, i.e., the dynamics of the timereversed series $X(-t)$.

If we assume dynamics of the form (1), we obtain the estimated driving noise $\tilde{\xi}(t)$, Eq. (4). The probability distribution of $\tilde{\xi}(t)$ would have lots of entries at zero if the dynamics was indeed following a jump-and-relax scheme and the original noise was not continuous. If the dynamics followed a building-up-and-reset scheme $\tilde{\xi}(t)$ would be fundamentally different. We can show this effect by looking at generalized $\mathrm{AR}(1)$ processes with different values for $p$. We will show this effect by looking at generalized AR(1) processes with different values for $p$ in Sec. III.B.

\section{A. Inferring the AR parameter}

The autoregressive parameter $g$ can be estimated in different ways. Inference by minimizing the squared residuals [Eq. (4)] yields a correct short time result but gives no validation for whether or not the obtained model describes the 
data well. Real data usually deviate from ideal AR(1) dynamics. Therefore, the timescale on which the exponent is fitted matters.

Using the autocorrelation function

$$
C(\Delta)=\frac{\langle X(t) X(t+\Delta)\rangle}{\sigma^{2}}
$$

still gives an estimation only for rather short times, but it offers some validation if the initial slope of the autocorrelation function indeed describes an exponential decay. It can then be fitted with a straight line on a semilogarithmic scale. The disadvantage of the autocorrelation function is that it cannot robustly be calculated for long lag times $\Delta$ because the function decays and fluctuates around zero without yielding information about its true behavior.

Another robust method is using the fluctuation function [15-17], which yields a model describing the dynamics on a wide range of timescales, thus producing model time series that "look" like the original recordings [18-20], although it does not necessarily yield the ideal values for the increments $X(t)-g X(t-1)$. One algorithm that can be used to calculate the fluctuation function $F(s)$ is the detrended fluctuation analysis [15]. An advantage of the method is that slow dynamical features like offsets or trends do not change the result due to detrending, which is implemented with a chosen order depending on a parameter $q$. For $q \leqslant 1$, offsets or asymmetries of the distribution (e.g., strictly positive values) do not affect the result. So the noise $\xi$ is not required to have zero mean. Traditionally the method was developed for inferring power law scaling of autocorrelations, however, here we use it in a different way.

The first step of the algorithm is to calculate the cumulative sum $y(t)=\sum_{n=1}^{t} x(n)$. In the second step the time series $y(t)$ is divided into $N$ nonoverlapping segments of length $s$. To each of these segments a polynomial $p_{n, s}^{q}(t)$ of order $q$ is fitted and subtracted. The fluctuations in the segment are quantified by the variance of this difference time series. In the third step, the fluctuation function is calculated as a function of segment length $s$ by averaging the $N$ segments with the same length

$$
F_{q}^{2}(s)=\left\langle\frac{1}{s} \sum_{t=1}^{s}\left\{y(t+(n-1) s)-p_{n, s}^{(q)}(t)\right\}^{2}\right\rangle_{n} .
$$

It describes the variability on a logarithmic scale (usually up to $s=1 / 4$ th of the length of the time series), which means that all timescales contribute equally. Therefore, the general structure of the data is visible and matching to data models is far simpler compared to other methods.

The empirical fluctuation function can be fitted to theoretical functions. They are given as transformations of the autocorrelation function [21]. The fluctuation function, which describes the exponential decay of autocorrelations [i.e., of $\mathrm{AR}(1)]$ for $q=1$ is given by

$$
F_{g}^{2}(s)=\sigma^{2} \frac{g^{s} J_{g}(s)+K_{g}(s)}{15(g-1)^{6}\left(s^{2}-s^{4}\right)},
$$

with $J_{g}(s), K_{g}(s)$ polynomials in $s$

$$
\begin{aligned}
J_{g}(s) & =60\left[s^{2}\left(g^{2}-g\right)^{2}-3 s\left(g^{3}-g\right)+2\left(g^{4}+g^{3}+g^{2}\right)\right], \\
K_{g}(s) & =s^{5}(g-1)^{5}(g+1)+15 s^{4} g(g-1)^{4}-5 s^{3} \\
& \times(g-1)^{3}\left(1-7 g-7 g^{2}+g^{3}\right)-15 s^{2} g(g-1)^{2} \\
& \times\left(1-10 g+g^{2}\right)+2 s\left(2-17 g-17 g^{2}+2 g^{3}\right) \\
& \times(g-1)^{3}-120 g^{2}\left(1+g+g^{2}\right) .
\end{aligned}
$$

The theoretical fluctuation function of $\operatorname{AR}(2)$ is known as well [17].

As stated above, this method might not be most accurate for minimizing $\tilde{\xi}(t)=X(t)-g X(t-1)$. However, it produces the best data model on a wide range of timescales. Therefore, it can be used for producing time-symmetric autoregressive models, against which the time asymmetry of the data can be tested in a hypothesis test.

\section{B. Dependence on the shape of the noise}

In Fig. 1 in the first column we show segments of time series, with different values for $p$, i.e., with different waiting times between the driving events. In the second column we show two estimations of $\xi$. One, $\tilde{\xi}_{+}=X(t)-$ $g X(t-1)$, assuming an AR(1) jump-and-relax dynamics and one, $\tilde{\xi}_{-}=X(t-1)-g X(t)$, assuming a building-upand-reset [time-reversed AR(1)] dynamics. We show the respective distributions for both versions of $\tilde{\xi}(t)$.

The two probability distributions of $\tilde{\xi}_{+}$and $\tilde{\xi}_{-}$are indistinguishable for the traditional $\operatorname{AR}(1)$ process $(p=1)$. If the increment distribution is no longer Gaussian due to waiting periods $(p<1)$, the distribution $\tilde{\xi}_{+}$correctly reproduces the driving noise with many values equal to zero. The distribution of $\tilde{\xi}_{-}$is very different.

A straightforward calculation of the variance of $\xi_{-}$using the iteration relation of the forward $\operatorname{AR}(1)$ process shows that it is identical to the one of $\xi_{+}$. So the two distributions have the same variance, but different higher moments. This can be measured in different ways. We propose the measure median $\left(\tilde{\xi}^{2}\right)$. This measure is shown for both $\tilde{\xi}_{+}$and $\tilde{\xi}_{-}$in the right column of Fig. 1. If the data are symmetric in time, both values are equal. We can therefore use the quotient

$$
\gamma=\frac{\operatorname{median}\left(\tilde{\xi}_{+}^{2}\right)}{\operatorname{median}\left(\tilde{\xi}_{-}^{2}\right)}
$$

The value indicates the type of dynamics

$$
\begin{array}{ll}
\gamma<1 & \text { jump-and-relax, } \\
\gamma=1 & \text { time-symmetric, } \\
\gamma>1 & \text { build-up-and-reset. }
\end{array}
$$

In Fig. 1 in the right column we show the calculated values of $\gamma$ for ensembles of all three processes with $p=1,2 / 3,1 / 3$.

\section{Dependence on the chosen AR parameter}

When analyzing real data, the process cannot be expected to perfectly follow the dynamics of the AR(1) model. Also, the parameter $g$ can only be estimated and is not known exactly as in the model. We, therefore, have to ask what a wrong choice of $g$ means for our method of identifying time-reversal asymmetry. 


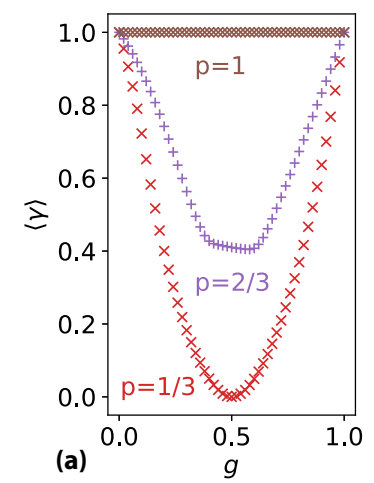

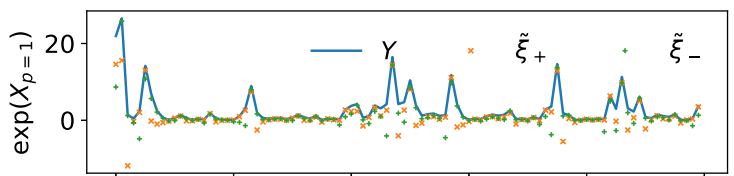

(b)

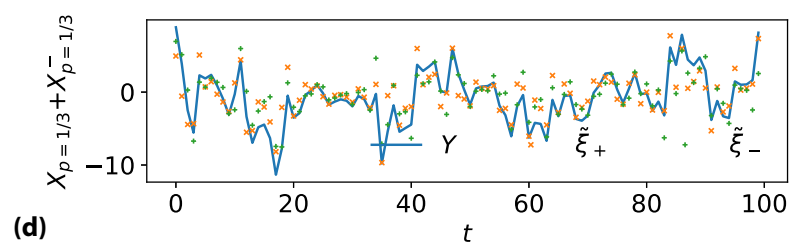

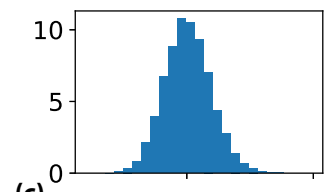

(c)

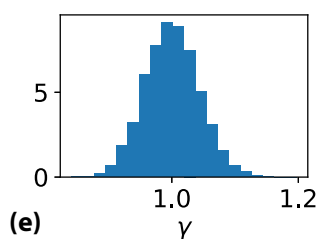

FIG. 2. (a) Ensemble averages (10 000 realizations) of $\gamma$ for generalized AR(1) processes with $g=0.5$. Reconstruction of $\tilde{\xi}_{ \pm}$with different (wrong) choices for parameter $g$ and the subsequent calculation of $\gamma$. Values close to the correct value $g=0.5$ give the largest deviation of $\gamma$ from 1. (b) Transformed Gaussian time series $Y=\exp (X)$, and reconstructed noise time series $\tilde{\xi}_{+}$(orange 'x') and $\tilde{\xi}_{-}$(green '+'). (c) The quotient $\gamma$ calculated from $\tilde{\xi}_{+}$and $\tilde{\xi}_{-}$of 10000 realizations of transformed Gaussian data yields values around 1, which shows that transformed Gaussian processes are time-reversal symmetric. (d) Superimposed timeseries with $p=1 / 3$ in both directions (original series $X_{p=1 / 3}$ and time-reversed series $X_{p=1 / 3}^{-}$), and reconstructed noise time series $\tilde{\xi}_{+}$(orange 'x') and $\tilde{\xi}_{-}$(green '+'). (e) The quotient $\gamma$ (distribution of 10000 realizations) shows that the superimposed process is time-reversal symmetric.

In Fig. 2(a) we show a numerical analysis of this issue. We generate ensembles of time series of generalized AR(1) processes with $g=0.5$ and calculate $\tilde{\xi}_{+}$and $\tilde{\xi}_{-}$for a range of values $g \in[0,1]$. The value $g=0$ trivially yields no difference between $\tilde{\xi}_{+}$and $\tilde{\xi}_{-}$because both time series are identical. For inserting $g=1$, the series just have opposite signs $\tilde{\xi}_{+}(t)=-\tilde{\xi}_{-}(t)$. In both cases, we get $\gamma=1$. For values of $g$ close to the correct value $g=0.5$, the deviations of $\gamma$ from 1 are maximal. The question is what happens for other values of $0<g<1$. In the figure, we see the behavior for $p=1,2 / 3$, and $1 / 3$. The closer the value of $g$ is to the ideal value, the stronger the deviation of $\gamma$ from 1 if the process is not time-symmetric $(p<1)$. The closer $g$ is to the trivial values 0 and 1 , the smaller the deviation of $\gamma$ from 1 becomes. So time asymmetry can be inferred even for wrong choices of $g$, however, the significance level rises around the correct value. One could also use the maximization of $|1-\gamma|$ to infer $g$, but one should notice that for a single time series only, a curve like in Fig. 2(a) has quite large fluctuations, so that this method does not lead to very precise estimates of $g$.

\section{Non-Gaussian yet time-symmetric data}

Due to the central limit theorem, Gaussianity is the most common assumption in time series analysis. When a measurement yields a non-Gaussian probability distribution, there might be different causes. The most common, but not the only, is nonstationarity of the probability distribution [22]. For stationary time series, either the system is truly non-Gaussian or the observable is a transformed version of a hidden Gaussian variable. Since Gaussian processes are time-symmetric, the time series would stay time-symmetric under a transformation. This is shown by our analysis of such a model system in Figs. 2(b) and 2(c). The obtained values for $\gamma$ in an ensemble lie around $\gamma=1$, which is the expected result.

Transformed Gaussian data are not the only example of non-Gaussian yet time-symmetric data. This is very easy to see by imagining the superposition of two time-asymmetric series with opposing direction (one jump-and-relax and one build-up-and-reset) and $\gamma_{1} \times \gamma_{2}=1$. We show such a superposition of two generalized $\operatorname{AR}(1)$ trajectories with $p=1 / 3$, along with the reconstructed driving noises $\tilde{\xi}_{+}$and $\tilde{\xi}_{-}$in Fig. 2(d). In Fig. 2(e), we show the histogram of numerically calculated values for $\gamma$ for an ensemble of such trajectories.

Thus we see, that non-Gaussianity does not imply timeasymmetry. The equivalence is only given for linear models as described above.

\section{APPLICATION TO REAL DATA}

We want to apply our framework to real systems and infer new information from the data. The applicability of exponential relaxations is very wide, as our selection of examples shows. We investigate stratospheric aerosol optical depth with long waiting periods between events and concentrations of the oxygen isotope $\delta^{18} \mathrm{O}$ in ice cores.

\section{A. Dansgaard-Oeschger events}

During the last glacial period, there were several large and rapid temperature fluctuations [23], i.e., short warm periods, which are known as Dansgaard-Oeschger events [24]. They are found from the concentrations of the oxygen isotope $\delta^{18} O$ in Greenland ice cores. The series constructed from these concentrations can be regarded as a temperature proxy over time. The origin of these events is still subject to discussion and there is a lot of literature about the timing of the events and suspected periodicities [25]. Here, we want to investigate the time asymmetry of the series, which is evident from visual inspection. We want to determine the result of our proposed method for this dataset.

In Fig. 3(a), we show the time series of $\delta^{18} O$ concentrations (NGRIP [26]) from $22090 \mathrm{bp}$ to $77840 \mathrm{bp}$ with a resolution of 50 years. Note, that the time order is reversed, as it is the convention in paleoclimate science. The sharper edges of the peaks are towards earlier times, while the younger side of some peaks seems to decay slower. The distribution of the series is clearly asymmetric. Therefore, the first task to 

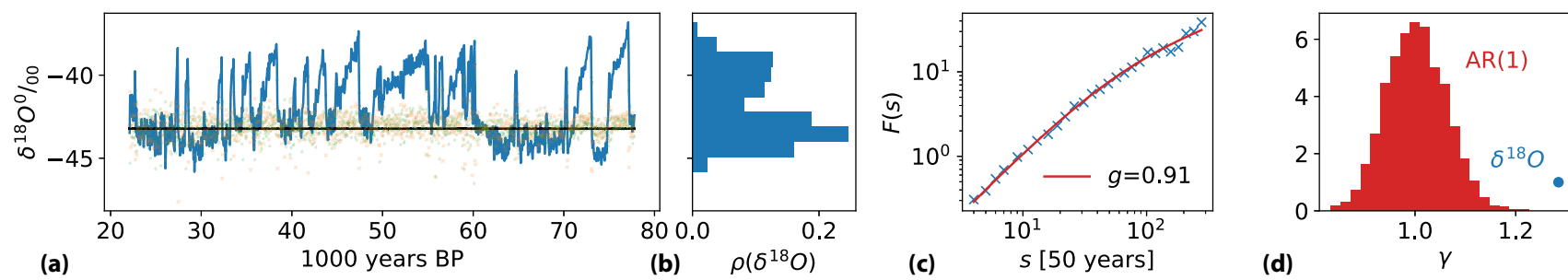

FIG. 3. (a) Temperature proxy from four ice cores $X$ (blue line), and reconstructed noise time series $\tilde{X}_{+}$(orange 'x') and $\tilde{X}_{-}$(green '+'). (b) Histogram of $X$. (c) DFA fluctuation function of $X(t)$ and fitted theoretical fluctuation function. The fitted parameter is $g=0.91$. (d) The quotient $\gamma$ calculated from $\tilde{X}_{+}$and $\tilde{X}_{-}$compared to the same observable for an ensemble of AR(1) processes with the same parameter $g=0.91$.

be done is to find the maximum of the distribution, i.e., the "zero" of the autoregressive process, to which the system decays if the driving is turned off. Note, that defining the "zero" in this way allows the distribution of the noise $\xi$ to have nonzero mean. A simple algorithm that solves the problem is to iteratively neglect the point with the largest distance to the mean value. By doing so, in each step, the mean value changes and gets shifted towards the maximum of the distribution. This algorithm works since the distribution [Fig. 3(b)] has a strong maximum. For a symmetric distribution, the obtained value coincides with the mean and median. For asymmetric distributions, median and mean clearly yield an offset, which is undesired in our case. The obtained "zero" is highlighted in Fig. 3(a).

The data are reasonably well described by a generalized AR(1) model. The parameter is determined to be $g=0.91$ by fitting the fluctuation function [see Fig. 3(c)]. The suspected periodicities [25] are not sufficiently pronounced to be visible in the fluctuation function (as previously reported [27]).

Since the offset and the parameter $g$ are now known, we can calculate the reconstructed noise terms $\tilde{\xi}_{+}$and $\tilde{\xi}_{-}$, the respective medians, and the quotient $\gamma$. The quotient is $\gamma=1.28$, which corresponds to build-up-and-reset dynamics in the series (which is reversed in time). To check the significance of this result, we calculate $\gamma$ for an ensemble of Gaussian AR(1) processes with the same variance $g$ and length as the $\delta^{18} O$ concentrations. The result in Fig. 3(d) shows that the result is in fact significant and the method concludes that the time series is asymmetric.

\section{B. Volcanic eruptions}

The time series of stratospheric aerosol optical depth [28] shows the impact of volcanic eruptions. It is one of the major climate forcings [29] for global temperature. The dynamics is intermittent. Spikes are caused by volcanic eruptions and decay slowly. The time series is presented in Fig. 4(a). Its distribution with a maximum close to zeros is shown in Fig. 4(b).

The decay has a very clear shape. We define a relaxation period as the interval between a local maximum and all following consecutively decreasing numbers. We define local maxima as values, which are larger than the previous value and the three following values $X_{\max }(t)>\max [X(t-$ $1), X(t+1), X(t+2), X(t+3)]$. The identified relaxation periods are highlighted in gray in the figure. In Fig. 4(c), we plot all relaxation periods on a semilogarithmic scale. This shows very clearly the exponential decay with a unique expo- nent $\beta$. This exponential decay corresponds to the dynamics of a generalized AR(1) model. The parameter $g$ is related to the exponent $\beta$ via $g=\exp (\beta)$.

Therefore, we can interpret the dynamics as a generalized $\operatorname{AR}(1)$ process and reconstruct the driving noise $\tilde{\xi}_{+}$. The quotient of the medians of squared noises is $\gamma=0.096$, indicating strongly asymmetric jump-and-relax dynamics [see Fig. 4(g)]. This is in line with the visual observation. Even though this result is clearly significant, we would like to design a proper hypothesis test against a symmetric model. Proper analysis of the fluctuation function of these data suggests using an $\mathrm{AR}(2)$. Fitting $g$ of an AR(1) process to such model data and calculating $\gamma$ from an ensemble of this process, we can prove the significance of the asymmetric jump-and-relax dynamics.

When looking at the fluctuation function [see Fig. 4(e)] of stratospheric aerosol optical depth, we see strong deviations from the $\mathrm{AR}(1)$ model. At first sight, this is surprising as it contradicts the clear exponential-relaxation dynamics we described. The deviation from the generalized AR(1) dynamics manifests itself in the building-up, before the volcanic eruption. We define them in the same way as we defined the relaxation periods, just for the time-reversed series. In Fig. 4(d) we show the building-up periods on a semilogarithmic scale. Unlike the relaxations, they do not have a universal exponential shape, however, a mean exponent can be fitted. We get $\beta=-0.075$. This exponent can also be found from the fluctuation function [see Fig. 4(f)] of the reconstructed noise $\tilde{\xi}_{+}$found above. So both the building-up and the relaxation can each be described by a generalized AR(1) model in the corresponding direction. An approximate model is given by feeding the output of one generalized AR(1) model as noise term into the other one.

\section{CONCLUSION}

We introduce a theory of time-reversal symmetry based on autoregressive processes. We distinguish time-symmetric dynamics from building-up-and-reset and jump-and-relax dynamics. We compare reconstructed increments of a linear response model $[\mathrm{AR}(1)]$. Theoretically, such a process is only time-symmetric if the distribution is Gaussian. However, since real data are not perfectly described by the model, Gaussianity and time symmetry are not at all equivalent. Time symmetry can also be explained by a transformed Gaussian variable or by heterogeneous events, i.e., a mixture of building-up-andreset and jump-and-relax dynamics. 

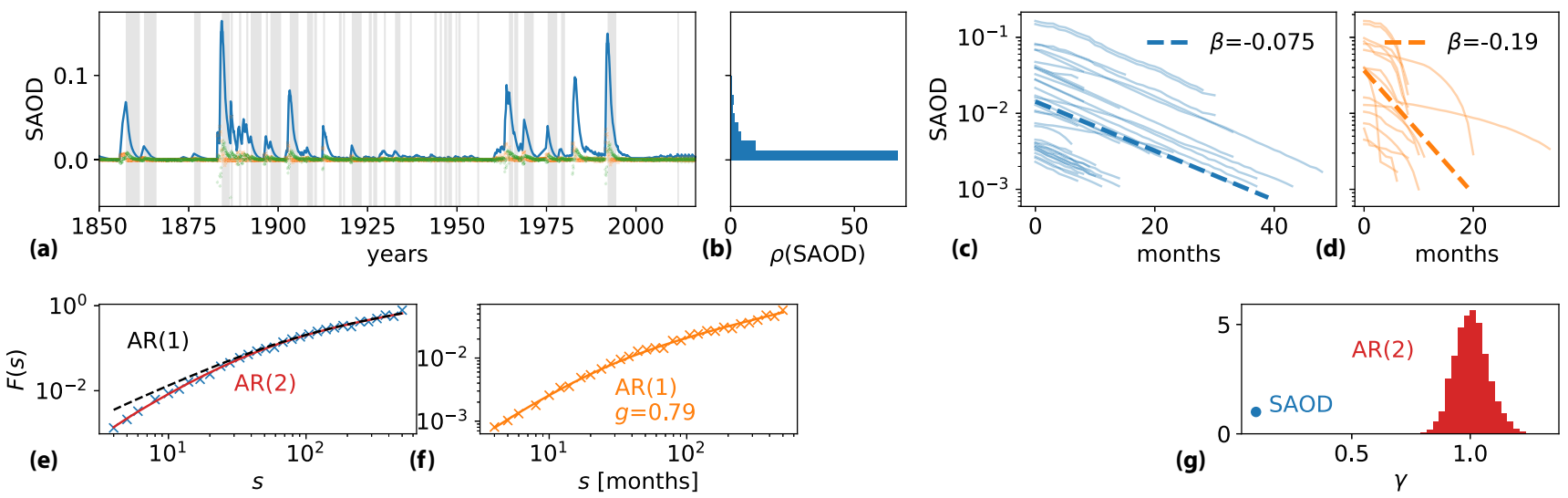

FIG. 4. (a) SAOD time series $X$ (blue line), and reconstructed noise time series $\tilde{\xi}_{+}$(orange 'x') and $\tilde{\xi}_{-}$(green '+'). (b) Histogram of $X$. (c) Trajectories of relaxations after volcanic eruptions; (dashed line) average exponential decay $\beta$. (c) Trajectories of building up before volcanic eruptions; (dashed line) average exponential decay $\beta$. (e) DFA fluctuation function of SAOD fitted by an AR(2) fluctuation function and an AR(1) fluctuation function, which strongly deviates. (f) DFA fluctuation function of $\tilde{\xi}_{+}$fitted by AR(1). (g) The quotient $\gamma$ calculated from $\tilde{\xi}_{+}$and $\tilde{\xi}_{-}$of SAOD compared to the same observable for an ensemble of AR(1) processes with exponent $\beta$.

There are different ways of how to infer the correct AR(1) model from data. For lag one a direct calculation from the $\mathrm{AR}(1)$ model is possible, fitting the autocorrelation function is more robust for short to intermediate timescales, for the most robust extraction on a wide range of scales we propose the fit the fluctuation function. This is especially useful for generating corresponding symmetric autoregressive models for hypothesis tests of asymmetry in the dataset. We test our approach with model data and show the sensitivity of the method depending on how well the $\mathrm{AR}(1)$ parameter is estimated. The method is robust if the time series is sufficiently asymmetric. Then we apply it to two measured time series from atmospheric science.

One example is North Greenland Ice Core Project (NGRIP) $\delta^{18} \mathrm{O}$ data during ice ages. The concentration is a temperature proxy over time. The series is characterized by short events of warm temperature (Dansgaard-Oeschger events). These are asymmetric spikes with nonuniform pacing. We show that the data can be approximated by a generalized AR(1) model. Our method yields asymmetric building-up-and-reset dynamics for the time-reversed dataset.

The recordings of stratospheric aerosol optical depth exhibit intermittent behavior. The waiting periods enable us to see the exponential decay explicitly. The time-reversal asymmetry is visible by the eye. Even though the system is not completely described by an AR(1) model and includes a second characteristic timescale, we apply our approach of reconstructing the noise under the assumption of $\mathrm{AR}(1)$ in both directions in time. The method yields strong time-reversal asymmetry.
[1] B. Øksendal, Stochastic differential equations, in Stochastic Differential Equations (Springer, New York, 2003), p. 11.

[2] G. Zumbach, Time reversal invariance in finance, Quantitative Finance 9, 505 (2009).

[3] Y. Pomeau, Symétrie des fluctuations dans le renversement du temps, J. Phys. 43, 859 (1982).

[4] J. H. Martínez, J. L. Herrera-Diestra, and M. Chavez, Detection of time reversibility in time series by ordinal patterns analysis, Chaos: An Interdisciplinary J. Nonlinear Sci. 28, 123111 (2018).

[5] J. S. W. Lamb and J. A. G. Roberts, Time-reversal symmetry in dynamical systems: A survey, Physica D 112, 1 (1998).

[6] C. Diks, J. C. Van Houwelingen, F. Takens, and J. DeGoede, Reversibility as a criterion for discriminating time series, Phys. Lett. A 201, 221 (1995).

[7] T. Schreiber and A. Schmitz, Improved Surrogate Data for Nonlinearity Tests, Phys. Rev. Lett. 77, 635 (1996).

[8] D. Kugiumtzis, On the reliability of the surrogate data test for nonlinearity in the analysis of noisy time series, Inte. J. Bifurcation Chaos 11, 1881 (2001).
[9] M. Dellnitz, G. Froyland, and S. Sertl, On the isolated spectrum of the perron-frobenius operator, Nonlinearity 13, 1171 (2000).

[10] G. E. P. Box, G. M. Jenkins, G. C. Reinsel, and G. M. Ljung, Time Series Analysis: Forecasting and Control (John Wiley \& Sons, New York, 2015).

[11] D. Maraun, H. W. Rust, and J. Timmer, Tempting longmemory-on the interpretation of dfa results, Nonlinear processes in Geophysics 11, 495 (2004).

[12] S. Hallerberg, E. G. Altmann, D. Holstein, and H. Kantz, Precursors of extreme increments, Phys. Rev. E 75, 016706 (2007).

[13] F. Corsi, A simple approximate long-memory model of realized volatility, J. Fina. Econometrics 7, 174 (2009).

[14] C. Junsheng, Yu. Dejie, and Y. Yu, A fault diagnosis approach for roller bearings based on emd method and ar model, Mechanical Systems and Signal Processing 20, 350 (2006).

[15] C. K. Peng, S. V. Buldyrev, S. Havlin, M. Simons, H. E. Stanley, and A. L. Goldberger, Mosaic organization of dna nucleotides, Phys. Rev. E 49, 1685 (1994). 
[16] M. Hoell and H. Kantz, The fluctuation function of the detrended fluctuation analysis - investigation on the AR(1) process, Eur. Phys. J. B 88, 126 (2015).

[17] P. G. Meyer and H. Kantz, Inferring characteristic timescales from the effect of autoregressive dynamics on detrended fluctuation analysis, New J. Phys. 21, 033022 (2019).

[18] P. G. Meyer and H. Kantz, A simple decomposition of european temperature variability capturing the variance from days to a decade, in Climate Dynamics (Springer, New York, 2019), pp. 1-9.

[19] P. G. Meyer, M. Anvari, and H. Kantz, Identifying characteristic time scales in power grid frequency fluctuations with DFA, Chaos 30, 013130 (2020).

[20] P. G. Meyer, H. Kantz, and Yu. Zhou, Characterizing variability and predictability for air pollutants with stochastic models, Chaos: An Inte. J. Nonlinear Sci. 31, 033148 (2021).

[21] M. Hoell and H. Kantz, The relationship between the detrendend fluctuation analysis and the autocorrelation function of a signal, Eur. Phys. J. B 88, 327 (2015).

[22] C. Beck and E. G. D. Cohen, Superstatistics, Physica A: Statistical mechanics and its applications 322, 267 (2003).

[23] W. Dansgaard, S. J. Johnsen, H. B. Clausen, D. Dahl-Jensen, N. S. Gundestrup, C. U. Hammer, C. S. Hvidberg, J. P.
Steffensen, A. E. Sveinbjörnsdottir, J. Jouzel et al., Evidence for general instability of past climate from a 250-kyr ice-core record, Nature 364, 218 (1993).

[24] P. D. Ditlevsen, M. S. Kristensen, and K. K. Andersen, The recurrence time of dansgaard-oeschger events and limits on the possible periodic component, J. Clim. 18, 2594 (2005).

[25] M. Schulz, On the 1470-year pacing of DansgaardOeschger warm events, Paleoceanography 17, 4-1 (2002).

[26] North Greenland Ice Core Project members et al., High resolution record of northern hemisphere climate extending into the last interglacial period, Nature 431, 147 (2004).

[27] Z.-G. Shao and P. D. Ditlevsen, Contrasting scaling properties of interglacial and glacial climates, Nat. Commun. 7, 10951 (2016).

[28] M. Sato, J. E. Hansen, M. Patrick McCormick, and J. B. Pollack, Stratospheric aerosol optical depths, 1850-1990, J. Geophys. Res. 98, 22987 (1993).

[29] J. Hansen, M. K. I. Sato, R. Ruedy, L. Nazarenko, A. Lacis, G. A. Schmidt, G. Russell, I. Aleinov, M. Bauer, S. Bauer et al., Efficacy of climate forcings, J. Geophys. Res. 110, D18104 (2005). 